\title{
Tunable picosecond infrared laser system based on parametric amplification in KTP with a Ti:sapphire amplifier
}

\author{
D. E. Gragson, D. S. Alavi, and G. L. Richmond \\ Department of Chemistry, University of Oregon, Eugene, Oregon 97403
}

Received May 25, 1995

\begin{abstract}
A picosecond laser system that will generate high-power tunable IR pulses with bandwidths suitable for spectroscopic applications is discussed. The system is based on white-light continuum generation in ethylene glycol and optical parametric amplification in potassium titanyl phosphate. The nonlinear-optical processes are driven by a regeneratively amplified Ti:sapphire laser that produces 1.7-ps pulses at a repetition rate of $1 \mathrm{kHz}$. Energies as high as 40 and $12 \mu \mathrm{J}$ have been achieved over the signal (1.02-1.16- $\mu \mathrm{m})$ and idler $(2.6-$ $3.7-\mu \mathrm{m})$ tuning ranges, respectively. The IR beam temporal and spatial characteristics are also presented. (C) 1995 Optical Society of America
\end{abstract}

Nonlinear-optical methods such as second-harmonic generation and sum-frequency generation are uniquely suited to the study of buried interfaces. In the case of visible/IR sum-frequency generation one can achieve both molecular and interfacial selectivity. Because of this unique selectivity the use of sum-frequency generation as a spectroscopic tool to study interfaces at a molecular level has grown rapidly over the past several years. ${ }^{1-3}$ One difficulty encountered in sumfrequency generation studies of molecular systems has been the lack of short-pulse, high-power tunable IR laser systems that can be used to probe the vibrational modes of condensed phase interfacial molecular species. Since the ability to resolve closely spaced vibrations directly depends on the spectral extent of the IR pulses, the bandwidths of the pulses also become a major consideration. Ti:sapphire systems show promise for nonlinear spectroscopic experiments, which benefit from the short pulses, and thus the high peak powers, that these lasers provide. However, for the majority of cases the short pulses do not have the narrow bandwidth necessary for spectral resolution of vibrational modes. In this Letter we describe a Ti:sapphire-based laser system that is optimized for providing both high peak powers and relatively narrow bandwidths. It consists of a Ti:sapphire regenerative amplifier that we use to generate high-power picosecond IR laser pulses tunable from 1 to $3.8 \mu \mathrm{m}$.

Parametric processes such as optical parametric generation and amplification in nonlinear crystals have been used extensively to generate tunable IR pulses. ${ }^{4,5}$ The majority of these systems are based on Nd:YAG pump lasers and produce pulses with durations from tens of picoseconds to nanoseconds ${ }^{6-8}$ and bandwidths from several wave numbers to a few tenths of a wave number. More recently regeneratively amplified Ti:sapphire lasers have been used to produce subpicosecond tunable IR pulses ${ }^{9-11}$ with bandwidths of the order of hundreds of wave numbers. We employ a Ti:sapphire regenerative amplifier that produces $1.7-\mathrm{ps}, 21-\mathrm{cm}^{-1}$ pulses, by spectral filtering in the stretcher, at $800 \mathrm{~nm}$ as our pump source. The IR generation is based on whitelight continuum generation in ethylene glycol and optical parametric amplification in two independently pumped potassium titanyl phosphate (KTP) crystals $(5 \mathrm{~mm} \times 5 \mathrm{~mm} \times 5 \mathrm{~mm})$. White-light generation was chosen to provide an intense seed covering the entire signal wavelength region, $1-1.6 \mu \mathrm{m}$. Our first attempt at IR generation involved seeding the first KTP crystal with the entire IR portion of the continuum from 1 to $1.6 \mu \mathrm{m}$. This, however, produced signal and idler bandwidths in excess of $100 \mathrm{~cm}^{-1}$, the large bandwidth being a result of the acceptance bandwidth of KTP, which is quite large in the $2.6-3.8-\mu \mathrm{m}$ region. One can narrow the bandwidth by supplying the KTP crystal with a narrower seed. This was accomplished by use of a grating to spatially disperse the continuum, resulting in a seed bandwidth of approximately $9 \mathrm{~cm}^{-1}$. KTP was selected as the nonlinear crystal because of the desired tuning range $(1-4 \mu \mathrm{m})$, its figure of merit (which is proportional to the conversion efficiency), and its availability. Further, type II (ordinary idler, extraordinary signal, ordinary pump) phase matching in the $x z$ plane of the KTP crystals was chosen because of the higher ${ }^{12} d_{\text {eff }}$ and larger tuning range for an $800-\mathrm{nm}$ pump. We use two amplification stages mainly because higher energies can be achieved but also because a second crystal angle tuned in the opposite direction has the effect of compensating for the beam displacement from the first crystal.

The Ti:sapphire regenerative amplifier laser system consists of a Ti:sapphire oscillator (Coherent Mira-900 basic) pumped by an $\mathrm{Ar}^{+}$-ion laser (Coherent Innova-310), a stretcher-compressor (Quantronix 4822), and a Ti:sapphire regenerative amplifier (Quantronix 4810) pumped by the second harmonic of a Nd:YLF laser (Quantronix $527 \mathrm{DP}-\mathrm{H}$ ). The output of the laser system $(750 \mu \mathrm{J}, 800 \mathrm{~nm}$, and 1-kHz repetition rate) is first split with an $80 \%$ reflective mirror that sends $600 \mu \mathrm{J}$ of energy to the optics for nonlinear conversion. Figure 1 depicts the optical setup used to convert the $800-\mathrm{nm}$ photons into IR photons. The $600-\mu \mathrm{J}$ beam is split into three pump lines with two $50 \%$ reflecting mirrors, BS1 and BS2. The three lines are used for pumping the white-light generator WL and the two KTP crystals, OPA1 and OPA2. Waveplate-polarizer combinations ( $\mathrm{H} 1-\mathrm{H} 3$ and $\mathrm{P} 1-\mathrm{P} 3)$ are used to set the polarization and energy on each 


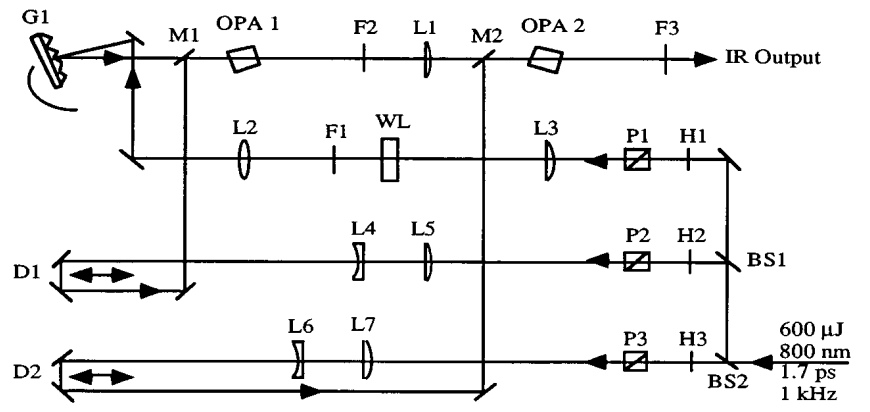

Fig. 1. Diagram of the optical parametric amplification system.

beam line. The white-light pump passes through lens L3, which focuses $125 \mu \mathrm{J}$ of the 800-nm light into the ethylene glycol. The ethylene glycol flows through a $10 \mathrm{~mm} \times 10 \mathrm{~mm} \times 50 \mathrm{~mm}$ quartz cell (WL) that limits the effect of bubble formation on the white-light generated by boiling. The effect of bubble formation is also minimized by cooling the ethylene glycol to approximately $16{ }^{\circ} \mathrm{C}$. The IR portion of the continuum is brought to a focus at the position of OPA1 by lens L2. The bandwidth of the seed supplied to OPA1 is narrowed by grating G1 (600 grooves $/ \mathrm{mm}$, blazed at $750 \mathrm{~nm}$ ) located $400 \mathrm{~mm}$ from OPA1. The white-light seed is then amplified in OPA1 with $145 \mu \mathrm{J}$ of the $800-\mathrm{nm}$ light focused to a beam diameter $2 \omega$ of $440 \mu \mathrm{m}$ $\left(56 \mathrm{GW} / \mathrm{cm}^{2}\right)$ by lenses L4 and L5. The IR seed and the $800-\mathrm{nm}$ pump are combined with dichroic mirror M1, and the temporal overlap of the seed and the pump in OPA1 is controlled by delay line D1. After this first stage of amplification signal energies as high as $20 \mu \mathrm{J}$ and idler energies as high as $4 \mu \mathrm{J}$ are obtained. Because higher energies are desired, another amplification stage (OPA2) located $560 \mathrm{~mm}$ from OPA1 is employed. Filter F2 (75\% transmission at $1.7-3.4 \mu \mathrm{m}$ ) is used to transmit the idler for amplification in OPA2. The idler is used for the seed in OPA2 because higher energies and better spatial beam profiles are attained in this configuration (see the discussion below). The idler from OPA1 is focused to a beam diameter $2 \omega$ of $760 \mu \mathrm{m}$ at the position of OPA2 by a $\mathrm{CaF}_{2}$ lens, L1. The idler generated in OPA1 is amplified in OPA2 with $200 \mu \mathrm{J}$ of the $800-\mathrm{nm}$ light, which is focused to a beam diameter $2 \omega$ of $600 \mu \mathrm{m}$ $\left(41 \mathrm{GW} / \mathrm{cm}^{2}\right)$ by lenses L6 and L7. The idler and the 800-nm pump are combined with the use of dichroic mirror M2, and the temporal overlap of the seed and the pump in OPA2 is controlled by a second delay line, D2. The pump beam diameters and energies were chosen such that the power density is below the damage threshold (approximately $80 \mathrm{GW} / \mathrm{cm}^{2}$ ) and the gain is not yet in the saturation regime.

The output of the laser system was characterized in terms of wavelength, power, bandwidth, pulse duration, and spatial beam quality. Wavelengths are measured with a $0.3-\mathrm{m}$ crossed Czerny-Turner monochromator with a $300-$ groove $/ \mathrm{mm}$ grating and an InSb detector. Bandwidths (FWHM) were measured with the monochromator or with a grating (1200 or 1800 grooves $/ \mathrm{mm}$ ) in combination with a one-dimensional CCD array (UniData BP2048). The resolution with the monochromator was approximately
$1 \mathrm{~cm}^{-1}$, whereas the resolution of the grating/CCD combination was approximately $4 \mathrm{~cm}^{-1}$ and depended on the diameter of the beam used. Powers were measured with a pyroelectric joulemeter, and pulse durations characterized by autocorrelation (Inrad 5-14B) and/or cross-correlation widths (FWHM). The spatial beam quality was characterized with knife-edge measurements across the horizontal transverse profile of the beams. The measured pump and idler beam sizes were then used to estimate the $M^{2}$ parameter for the idler beam.

Theoretical tuning curves were generated by imposing type II (oeo) collinear phase matching in the $x z$ plane $\left(\phi=0^{\circ}\right)$ of the KTP crystal. The Sellmeier constants used are from Vanherzeele et al. ${ }^{12}$ Both the theoretical and the experimental tuning curves over a practical tuning range are shown in Fig. 2. A practical tuning range is limited by relative misalignment of the pump and seed beams in the KTP crystals that occurs as they are rotated. With intermediate realignment, the actual tuning curve extends from beyond 3.8 to $1.6 \mu \mathrm{m}$ for the idler and from 1.6 to $1.01 \mu \mathrm{m}$ for the signal.

We generated the tuning curves in Fig. 2 by angle tuning both the KTP crystals and the grating. As was mentioned above, the bandwidth of the output IR pulses when seeded with the entire IR portion of the continuum is greater than $100 \mathrm{~cm}^{-1}$. By use of a diffraction grating so that the first-order diffracted beam is propagated through the optical parametric amplifier, the bandwidth of the amplified signal beam was reduced to approximately $31 \mathrm{~cm}^{-1}$. A rough calculation of the seed bandwidth expected to fall within the spatial profile of the pump beam in the first KTP crystal $(260 \mu \mathrm{m}$ FWHM) yields $\Delta \bar{\nu}=a \bar{\nu}^{2} \cos \beta(d / D)=8.6 \mathrm{~cm}^{-1}$, where $a$ is the groove spacing of the grating, $d$ is the diameter of the pump beam (FWHM), $D$ is the distance from the grating to the first KTP crystal, and $\beta$ is angle of diffraction from the grating. Since the bandwidth of the pump, $21 \mathrm{~cm}^{-1}$, is greater than the bandwidth of the seed, $8.6 \mathrm{~cm}^{-1}$, we expect that the pump bandwidth will be a lower limit for the signal and idler bandwidths. In fact, bandwidths of $25-32 \mathrm{~cm}^{-1}$ were

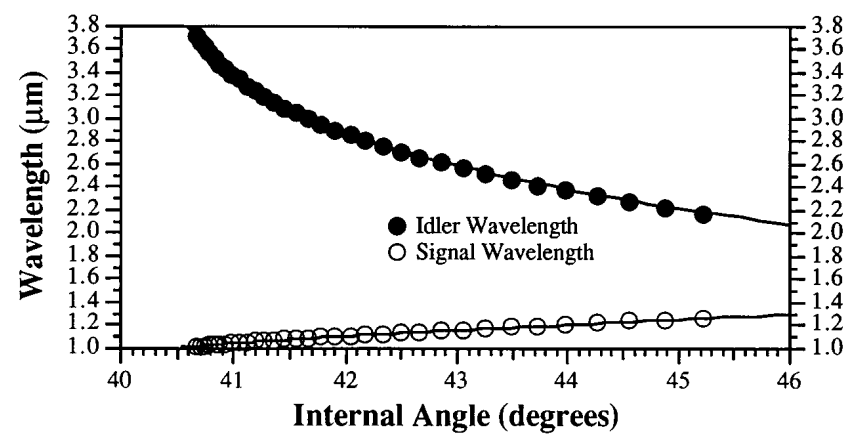

Fig. 2. Experimental and theoretical tuning curves for type II ( $o$, idler; $e$, signal; $o$, pump) phase matching in KTP. The open circles are the experimental data for the signal beam, and the filled circles are the experimental data for the idler beam. The solid curves are the theoretical tuning curves from the Sellmeier equations (see text). The KTP crystals are $5 \mathrm{~mm} \times 5 \mathrm{~mm} \times 5 \mathrm{~mm}$ and cut at $\theta=42.65^{\circ}$ and $\phi=0^{\circ}$. 


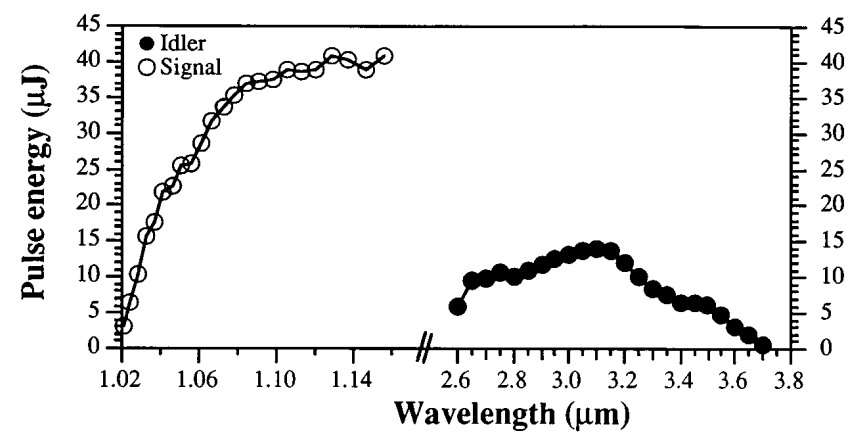

Fig. 3. Output energy of signal (open circles) and idler (filled circles) beams for the experimental tuning range in Fig. 2. Measured energies were corrected for filter losses, then plotted.

obtained throughout the tuning range shown in Fig. 2 for both signal and idler pulses.

The output from the first stage of parametric amplification consists of an amplified signal pulse and the corresponding idler pulse. Because of group-velocity mismatch effects, these pulses are not well overlapped temporally as they leave the first KTP crystal, so only one of these two pulses can be effectively amplified in the second KTP crystal. The idler was chosen for several reasons. First, the wavelength region of interest to us experimentally corresponds to the idler wavelengths, and seeding the second crystal with the idler up to $50 \%$ can yield more energy in the idler pulses than seeding with the signal. Second, it was observed that the output beam profile from the second KTP crystal was qualitatively better when it was seeded with the idler. When the output beam was seeded with the signal, the output of the second parametric amplifier stage was more divergent and had an elliptical transverse profile, possibly as a result of acceptance angle or acceptance bandwidth effects in the KTP crystal. This was not investigated further.

The propagation of the idler beam produced in the first KTP crystal was characterized by a series of knifeedge measurements. Assuming an idler beam waist of $\omega_{0}=220 \mu \mathrm{m}$ (the pump beam size) in the first KTP crystal yielded an estimate of $M^{2} \approx 2.5$. This beam was then focused by lens L1 (see Fig. 1) to a spot size of $\omega=380 \mu \mathrm{m}$ in the second KTP crystal. A series of knife-edge measurements was also made after the second KTP crystal to characterize the propagation of the final idler output beam. From these measurements a beam waist $\omega_{0}$ of $180 \mu \mathrm{m}$ and an $M^{2}$ value of 1.7 were obtained. No assumption of the beam waist was made in the determination of the beam-propagation parameters for the final idler beam.

A power curve was generated by rotation of the grating and the crystals together with no intervening realignment and is shown in Fig. 3. The idler pulse energies were greater than $5 \mu \mathrm{J}$ over a range of $2.7-3.5 \mu \mathrm{m}$ and greater than $10 \mu \mathrm{J}$ over $2.7-3.2 \mu \mathrm{m}$ and have a relative instability of $4 \%$ (standard deviation). It should be emphasized that we generated these curves by aligning the system once at the beginning and making no further adjustments as the wavelength was scanned by rotating the grating and KTP crystals. Another consideration is that the particular filters used in this configuration are not suitable for the entire tuning range. In particular, filter F2 has a nominal transmission range of $1.7-3.4 \mu \mathrm{m}$ and so begins to limit the amount of idler available to seed the second KTP crystal as the system is tuned beyond $3.5 \mu \mathrm{m}$. Filter F3 has a nominal transmission cutoff below $2.5 \mu \mathrm{m}$, limiting the amount of idler energy measurable below that wavelength.

The temporal and bandwidth characteristics of the pump, the signal, and the idler were measured. The output of the Ti:sapphire laser system has a bandwidth of $21 \mathrm{~cm}^{-1}$ and a pulse width of $1.7 \mathrm{ps}$ (2.2-ps FWHM autocorrelation), resulting in a time-bandwidth produce of 1.05, or 1.2 times transform limited (assuming sinc $^{2}$ pulses). These pulses were cross correlated with both signal and idler pulses by sum-frequency generation in another KTP crystal, yielding cross-correlation widths (FWHM) of 3.0 and $2.6 \mathrm{ps}$ for the signal and the idler pulses, respectively.

The intense IR pulses that are produced by this system make it an attractive source for nonlinear spectroscopic applications. In addition, the moderately narrow bandwidths and broad tuning range will allow for the study of a large number of condensed phase interfacial molecules through their vibrational modes. The $25-30-\mathrm{cm}^{-1}$ bandwidths reported here are well suited for studies of this type in which the bandwidths of the molecular vibrations are relatively large. However, if still narrower bandwidths are desired the pump laser source can be modified to give slightly longer pulses, which will result in a smaller bandwidth. The practical limit of the bandwidths attainable by this type of modification is approximately $10-15 \mathrm{~cm}^{-1}$. The use of other nonlinear crystals such as $\mathrm{AgGaS}_{2}$ and KTA to extend the tuning range beyond $3.8 \mu \mathrm{m}$ would also allow us to study a wider variety of molecules.

This work is supported by the U.S. Office of Naval Research.

\section{References}

1. R. Superfine, J. Y. Huang, and Y. R. Shen, Phys. Rev. Lett. 66, 1066 (1991).

2. Q. Du, E. Freysz, and Y. R. Shen, Science 264, 826 (1994).

3. D. Zhang, J. H. Gutow, T. F. Heinz, and K. B. Eisenthal, J. Chem. Phys. 98, 5099 (1993).

4. H. M. van Driel and G. Mak, Can. J. Phys. 71, 47 (1993).

5. R. Laenen, K. Wolfrum, A. Seilmeier, and A. Laubereau, J. Opt. Soc. Am. B 10, 2151 (1993).

6. H.-J. Krause and W. Daum, Appl. Phys. B 56, 8 (1993).

7. H. Vanherzeele, Appl. Opt. 29, 2246 (1990).

8. U. Sukowski and A. Seilmeier, Appl. Phys. B 50, 541 (1990).

9. F. Seifert, V. Petrov, and M. Woerner, Opt. Lett. 19, 2009 (1994).

10. S. Takeuchi and T. Kobayashi, J. Appl. Phys. 75, 2757 (1994).

11. P. Hamm, C. Lauterwasser, and W. Zinth, Opt. Lett. 18, 1943 (1993).

12. H. Vanherzeele, J. D. Bierlein, and F. C. Zumsteg, Appl. Opt. 27, 3314 (1988). 